\title{
Two-pulse control of Raman scattering in liquid methanol: The dominance of classical nonlinear optical effects
}

\author{
Michael Spanner and Paul Brumer \\ Chemical Physics Theory Group, Department of Chemistry, and Center for Quantum Information and Quantum Control, \\ University of Toronto, Toronto, Ontario, Canada M5S $3 H 6$ \\ (Received 23 September 2005; published 13 February 2006)
}

\begin{abstract}
Experimental results on adaptive feedback control of transient (i.e., nonimpulsive) Stokes emission in liquid methanol [Pearson and Bucksbaum, Phys. Rev. Lett. 92, 243003 (2004)] are analyzed. In the experiment, a pump pulse comprising two frequency-shifted Gaussian pulses was used to control the ratio of two Stokes emission lines by varying the relative phase $\phi_{L}$ between the pulses. Extending the theory of stimulated Raman scattering to accommodate two coupled levels, we show that control of this type is possible, in the strongly driven regime, using Raman coupling alone. Control via variation of $\phi_{L}$ is shown to also result from self- and cross-phase-modulation of the pump and Stokes pulses as well as via the focused-beam geometry of the pump pulse. In all cases, the general control mechanism is nonlinear optical modulation between the pump and the Stokes pulse; no coherent quantum interference effects are involved. Finally, although the vibrational populations are affected by the same control mechanisms that affect the Stokes spectra, the ratio of the Stokes spectra peak heights does not directly reflect the ratio of the level populations, as was assumed in the experiment.
\end{abstract}

DOI: 10.1103/PhysRevA.73.023810

\section{INTRODUCTION}

In an effort to understand seminal experiments on control of Stokes emission in liquids [1-5], we developed, in the preceding paper [6], a theory of two-mode Raman scattering of a broadband pump pulse. By tuning simple pulse parameters, such as the pulse duration and/or intensity, or by adding asymmetry to the spectral amplitudes across the bandwidth of the pulse, we were able to demonstrate control over the ratio of the Stokes emission arising from two closely spaced Raman modes, the observable in these experiments.

In this paper, we extend this treatment, focusing on a particular control experiment [5], where the relative peak heights of two closely spaced Stokes lines in the Raman emission of liquid methanol was controlled using only a double-Gaussian pump pulse. The pump pulse consisted of two frequency-shifted pulses with Gaussian envelopes in time, of duration $1 \mathrm{ps}$, with the frequency shift equal to the energy spacing between the two Raman modes. The resulting composite pulse can then be written as a "carrier pulse" of $1 \mathrm{ps}$ (centered at the mean frequency of the two subpulses) modulated by a cosine at the frequency of the mode spacing $\Delta$. The relative peak heights of the two Stokes lines was controlled by varying the relative phase $\phi_{L}$ between the two Gaussian components in the pump pulse, which shifts the phase of the cosine modulation relative to the carrier envelope. The use of a laser phase $\phi_{L}$ as a control variable suggests that this experiment may well be an example of liquidphase coherent control $[7,8]$, i.e., control via the quantum coherence of the incident light and matter. We show below that this is, in fact, not the case. Further, we demonstrate that the observed control is over the Stokes emission and that this does not imply control over the populations of the two associated methanol vibrational states, as previously proposed [5].

Specifically, we demonstrate below, using our theory of two-mode Raman scattering, that $\phi_{L}$-based control with Raman coupling alone is possible in the strongly driven regime where the pump pulse undergoes depletion, i.e., when the
PACS number(s): 42.65.Dr, 42.65.Re, 32.80.Qk

pump and Stokes pulses begin to undergo third-order nonlinear optical modulation (saturated stimulated Raman scattering [9]). Coherent excited-state population transfer between the excited vibrational states arises as part of the fifth-order nonlinear response of the system, and contributes negligibly to the experimental control of the Raman emission. This treatment corrects a previous incorrect analysis of these experiments $[5,10]$

Additional competing third-order nonlinear effects, such as self- and cross-phase-modulation [11], are likely to be present in the strongly driven regime. We found that these nonlinear propagation effects, acting on both the pump and Stokes pulses, also affect the relative peak heights in the stimulated Raman spectrum, as does the focused-beam geometry used in the experiment [5].

In all cases considered, a dependence of the Stokes spectrum on the relative phase $\phi_{L}$ between the two frequencyshifted Gaussian pump pulses was found to be possible only when the pump and Stokes pulses underwent nonlinear optical modulation. This means, for the double-Gaussian scenario, that the general control mechanisms are third-order nonlinear optical effects acting on the pump and Stokes pulses. Here, the third-order nonlinear control mechanisms explored are saturated Raman scattering, both with and without a focused-beam geometry, and Raman scattering in the presence of self- and cross-phase-modulation. $\phi_{L}$-dependent control over the Stokes emission is clearly demonstrated with all of the qualitative experimental features properly accounted for. However, precise agreement with the experimental results would require far more experimental data in order to quantify contributions from the numerous nonlinear optical effects.

\section{TWO-MODE RAMAN COUPLING}

\section{A. Formalism}

The relevant vibrational states of methanol are modeled by a three-level system shown in Fig. 1. The laser (pump) $\boldsymbol{E}_{L}$ 


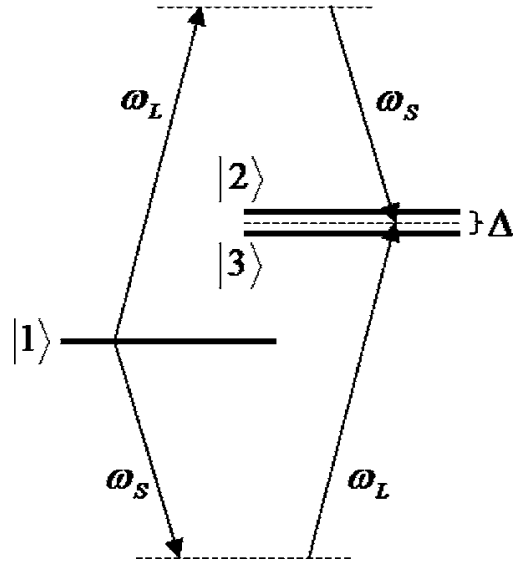

FIG. 1. The pump laser field at frequency $\omega_{L}$ drives Raman emission near frequency $\omega_{S}$ in a three-level system through twophoton Raman couplings. The two excited states are closely spaced with an small energy separation of $\Delta$ that is on the order of the bandwidth of the laser and Stokes pulses.

and Stokes $\boldsymbol{E}_{S}$ fields are far off resonant with the excited electronic states. We assume that the dipole transitions between the vibrational states of interest $|1\rangle,|2\rangle$, and $|3\rangle$ are negligible, where the latter two states are spaced by energy $\Delta$. (Note that all equations are written in atomic units where $\hbar=e=m_{e}=1$.) Transitions between these three states take place via two-photon Raman transitions through high-lying off-resonant states $|m\rangle$. The equations of motion coupling the laser and Stokes fields to the molecular vibrations are [6]

$$
\begin{aligned}
& \frac{\partial}{\partial z} E_{L}(z, t)=i \eta_{L} E_{S}(z, t)\left[\kappa_{21} Q_{21}(z, t) e^{-i(\Delta / 2) t}\right. \\
& \left.+\kappa_{31} Q_{31}(z, t) e^{i(\Delta / 2) t}\right] \\
& \frac{\partial}{\partial z} E_{S}(z, t)=i \eta_{S} E_{L}(z, t)\left[\kappa_{21} Q_{21}^{*}(z, t) e^{i(\Delta / 2) t}\right. \\
& \left.+\kappa_{31} Q_{31}^{*}(z, t) e^{-i(\Delta / 2) t}\right] \\
& \frac{\partial}{\partial t} Q_{21}(z, t)+\Gamma_{2} Q_{21}(z, t)=i \kappa_{21} E_{L}(z, t) E_{S}^{*}(z, t) e^{i(\Delta / 2) t}+\mathcal{F}_{2}, \\
& \frac{\partial}{\partial t} Q_{31}(z, t)+\Gamma_{3} Q_{31}(z, t)=i \kappa_{31} E_{L}(z, t) E_{S}^{*}(z, t) e^{-i(\Delta / 2) t}+\mathcal{F}_{3}, \\
& \begin{aligned}
\frac{\partial}{\partial t} Q_{22}(z, t)+\gamma_{2} Q_{22}(z, t)= & -i \kappa_{21} E_{L}^{*}(z, t) E_{S}(z, t) e^{-i(\Delta / 2) t} Q_{21}(z, t) \\
& + \text { c.c. },
\end{aligned} \\
& \frac{\partial}{\partial t} Q_{33}(z, t)+\gamma_{3} Q_{33}(z, t)=-i \kappa_{31} E_{L}^{*}(z, t) E_{S}(z, t) e^{i(\Delta / 2) t} Q_{31}(z, t) \\
& \text { + c.c., }
\end{aligned}
$$

where the electric fields are given by

$$
\begin{aligned}
& \boldsymbol{E}_{L}(z, t)=E_{L}(z, t) e^{-i \omega_{L} t+\overrightarrow{k_{L}} \cdot \vec{r}}+\text { c.c. }, \\
& \boldsymbol{E}_{S}(z, t)=E_{S}(z, t) e^{-i \omega_{S} t+\overrightarrow{k_{S}} \vec{r}}+\text { c.c. },
\end{aligned}
$$

with c.c. denoting the complex conjugate, $Q_{n m}(z, t)$ $=\rho_{n m}(z, t) e^{-i \omega_{n m} t}$ are the slowly varying envelopes of the molecular density matrix $\rho_{n m}(z, t)$ in the field-free basis, $\gamma_{i}$ is the decay rate of the excited-state populations, $\Gamma_{i}$ is the collision linewidth of the $i$ th Raman transitions that accounts for dephasing of the vibrational response, and the $\mathcal{F}_{i}$ terms represent the random Langevin force [12] due to collisions in the liquid. The constants $\kappa_{1 i}$ and $\eta_{n}(n=L, S)$ are

$$
\kappa_{1 i}=\sum_{m} \mu_{i m} \mu_{1 m}\left(\frac{1}{\omega_{m 1}-\omega_{L}}+\frac{1}{\omega_{m 1}+\omega_{S}}\right)
$$

and

$$
\eta_{n}=\frac{2 \pi N \omega_{n}}{c} \quad(n=L, S)
$$

where the $\mu_{i j}$ are the transition dipole matrix elements connecting the ground state $|1\rangle$ and Raman-active levels $|2\rangle$ and $|3\rangle$ to intermediate $|m\rangle$ states, and $N$ is the number density of molecules per unit volume.

Consider now the experimental Raman lasing scenario outlined in Ref. [5]. A pump pulse comprising two Gaussians shifted from one another in frequency by an amount equal to the excited-state energy separation $\Delta$ was used to drive the Stokes emission. Varying the relative phase between the two frequency-shifted Gaussians allowed control over the asymmetry in the peak heights of the two Stokes lines.

\section{B. Computational results}

To computationally examine this scenario, we consider an initial pump pulse shape given by

$$
E_{L}(z=0, t)=2 E_{0} \exp \left[-4 \ln 2\left(t / t_{p}\right)^{2}\right] \cos \left[(\Delta / 2) t+\phi_{L} / 2\right]
$$

where $t_{p}$ is the full width at half maximum of the Gaussian pulses, and $\phi_{L}$ is the relative phase (called the phase offset) between these two frequency-shifted Gaussians pulses. Below, we use $E_{0}=0.0017$ a.u., corresponding to an intensity of $10^{11} \mathrm{~W} / \mathrm{cm}^{2}$, and $t_{p}=1 \mathrm{ps}$, in accord with the experiments. Further, the experiments used a titanium:sapphire laser $(800 \mathrm{~nm})$ to generate the pump pulse. With Raman shifts of 2837 and $2947 \mathrm{~cm}^{-1}$ for the two Stokes lines [13], the central Stokes wavelength is then $1025 \mathrm{~nm}$, while the energy separation of the two Raman modes is $\Delta=110 \mathrm{~cm}^{-1}$. The linewidths of the Raman transition are $\Gamma_{2}=17 \mathrm{~cm}^{-1}$ and $\Gamma_{3}=34 \mathrm{~cm}^{-1}$ [13]. We assume that the decay times of the excited-state populations are much larger than $t_{p}$ so that $\gamma_{2} \simeq \gamma_{3} \simeq 0$. The two-photon coupling terms are chosen to be $\kappa_{31}=3.0$ a.u. and $\kappa_{21}=2.5$ a.u., values of $\kappa_{1 i}$ set to loosely mimic the experimental Stokes spectrum (ratio of peak heights and Stokes intensity relative to the pump pulse) driven by the unshaped pump pulse. The Stokes emission was seeded with the collisional terms $\mathcal{F}_{2}$ and $\mathcal{F}_{3}$, as outlined 

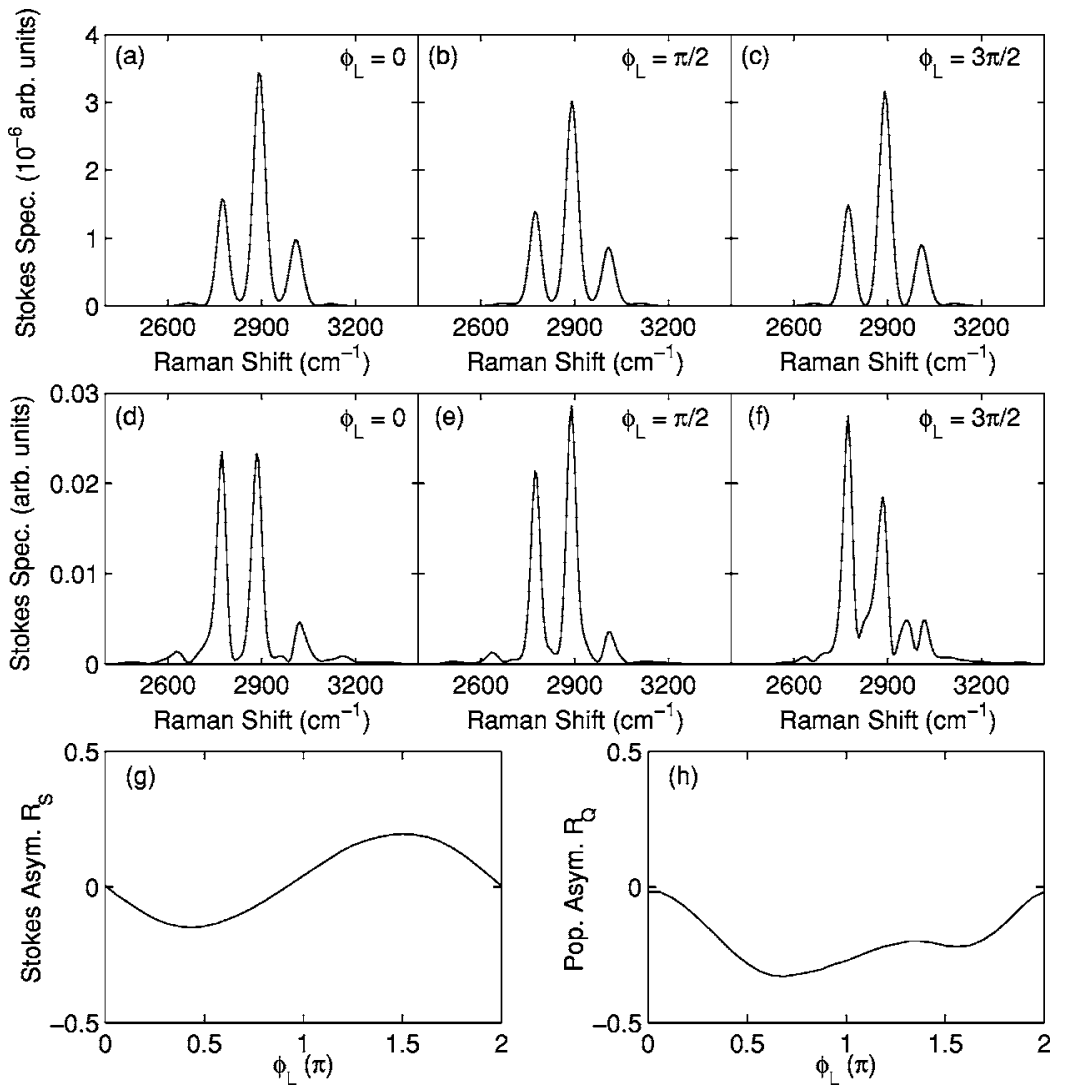

FIG. 2. Spectrum of the Stokes emission using the double-Gaussian pump pulse. The top row (a),(b),(c) shows Stokes spectra in the region of exponential gain $(z=0.05 \mathrm{~cm})$ for the three phase offsets $\phi_{L}=0, \pi / 2,3 \pi / 2$. The middle row (d),(e),(f) shows the Stokes spectra in the region of pump saturation $(z=0.15 \mathrm{~cm})$, again for the same three phase offsets. (g) shows the Stokes mode asymmetry $\mathcal{R}_{S}$ as a function of the phase offset, while (h) plots the population asymmetry $\mathcal{R}_{Q}$, both in the region of pump depletion. in the previous article [6]. Simulations that use spontaneous emission $[12,14]$ as a seeding term give qualitatively similar results as those shown throughout this paper, although for differently tuned parameters. Equations (1a) and (1b) were numerically integrated using Euler's method, while Eqs. (1c)-(1f) were treated with Verlet's method.

Figure 2 shows the resulting Stokes emission $\left|E_{S}(\omega)\right|^{2}$ using the double-Gaussian pump for a variety of relative phases $\phi_{L}=0, \pi / 2$, and $3 \pi / 2$. The top three panels (a)-(c) correspond to a propagation length of $z=0.05 \mathrm{~cm}$, and show the behavior of the Stokes spectra in the region of exponential gain, i.e., before the Stokes intensity has become comparable to the pump intensity. In this region, the Stokes spectrum shows a triple-peaked structure whose shape depends very little on the relative phase $\phi_{L}$. This triple-peaked structure can be deduced from simple energy considerations of the allowed Stokes emission. That is, the two central frequency components $\omega_{L 1}=\omega_{L}-\Delta / 2$ and $\omega_{L 2}=\omega_{L}+\Delta / 2$ in the pump pulse can each drive two central Stokes modes $\omega_{S}=\omega_{L 1 / L 2}-\omega_{21} \pm \Delta / 2$, giving a total of four central frequencies expected in the Stokes spectrum. However, since the separation of the two central frequencies in the pump pulse is the same as the separation of the two excited states, two of the four central Stokes frequencies are the same, resulting in the calculated triple-peaked structure. Since the middle peak is composed of the two degenerate frequency contributions, it larger than the remaining two nondegenerate peaks. These Stokes spectra, corresponding to the region of exponential Stokes gain, do not resemble the experimentally reported spectra, which exhibits two dominant peaks.

Figures 2(d)-2(f) show the calculated Stokes emission at $z=0.15 \mathrm{~cm}$, which is in the regime of pump saturation, i.e., when the intensity of the Stokes pulse has become comparable to the pump intensity. In this regime, the pump and Stokes pulses start to be strongly modulated through the large nonlinear response of the molecular medium. (This effect has been seen experimentally in the case of a single active Raman level driven by strong pump and Stokes pulses [9].) The Stokes emission has lost its triple-peaked structure, and now qualitatively resembles the experimental results [5], exhibiting two Stokes lines. Furthermore, the ratio of peak heights of the two Stokes lines now depends on the phase offset $\phi_{L}$, in accord with the control shown experimentally [5]. One can also see in Figs. 2(d)-2(f) the onset of multiplesideband generation in the Stokes emission, an effect that is qualitatively similar to the sideband generation created using a single Raman level and a strong pump pulse with two central frequency components whose energy separation is close to the level separation of the Raman transition [15]. Small additional sidebands can also be seen in the experimental spectra, supporting the view that the experimental spectra likely correspond to the strongly driven scenario.

There are three main differences between our simulated spectra Figs. 2(d)-2(f) and the experimental results in Ref. [5]. First, our peaks are narrower than the experimental Stokes spectrum, which shows two Stokes lines that overlap one another. The Raman coupling studied in this section, as well as the additional nonlinear propagation effects considered in the following sections, allow for frequency broadening. However, this broadening appears as regularly spaced sidebands. The broadening of the experimental Stokes lines may be due to nonuniform wave fronts of the shaped laser pulse, which could break the symmetry of the propagation equations, and cause continuous frequency broadening as op- 
posed to strict sideband generation. Second, our two dominant lines are shifted by $\Delta / 2$ from the standard positions 2837 and $2947 \mathrm{~cm}^{-1}$, unlike the experimental spectra [5]. Although it is clear why the shift appears in the simulated spectra (i.e., the two central lines in our pump pulse are shifted from the average carrier frequency by $\pm \Delta / 2$ ), it is not clear why a similar shift is not seen experimentally. The third discrepancy concerns the $\phi_{L}$ dependence of the ratio of the two peak heights. Figure $2(\mathrm{~g})$ plots the Stokes mode asymmetry $\mathcal{R}_{S}$ defined as

$$
\mathcal{R}_{S}=\frac{h_{L}-h_{R}}{h_{L}+h_{R}}
$$

as a function of $\phi_{L}$. Here $h_{R}$ and $h_{L}$ denote the heights of the right $(R)$ and left $(L)$ Stokes peaks, respectively. A value of $\mathcal{R}_{S}=1$ (or -1 ) indicates that all the intensity is in the left (or right) mode, while $\mathcal{R}_{S}=0$ indicates that the two lines are of equal intensity. The mode asymmetry is roughly sinusoidally dependent on $\phi_{L}$. The experiment report similar behaviour, but with $\mathcal{R}_{S} \propto \sin \left(2 \phi_{L}\right)$. Since the pump pulse is periodic in $\phi_{L}$, but not in $2 \phi_{L}$, we expect that our results are indeed correct.

The simulated Stokes spectrum was found to depend very sensitively on the particular system parameters (pump intensity, propagation length, two-photon couplings, etc.). Therefore, a large amount of flexibility in, and hence control over, the shape of the output Stokes spectrum, as well as the $\phi_{L}$ dependence of $\mathcal{R}_{S}$, exists.

Consider now the dynamics of the vibrational populations $Q_{22}$ and $Q_{33}$. Figure 2(h) shows the population asymmetry $\mathcal{R}_{Q}$, defined as

$$
\mathcal{R}_{Q}=\frac{Q_{22}\left(z, t=t_{f}\right)-Q_{33}\left(z, t=t_{f}\right)}{Q_{22}\left(z, t=t_{f}\right)+Q_{33}\left(z, t=t_{f}\right)},
$$

where $t=t_{f}$ denotes the end of the pump pulse. Comparing the population asymmetry $\mathcal{R}_{Q}$ to the Stokes asymmetry $\mathcal{R}_{S}$, it is clear that the final Stokes asymmetry does not reflect the ratio of populations. However, a large degree of variation of the population asymmetry is seen, even if it is not directly reflected in the Stokes spectra. This is in accord with our previous results [6], especially when the bandwidth of the pump pulse is of the same order as the Raman mode energy spacing.

Note that the $\phi_{L}$ dependence of both $\mathcal{R}_{S}$ and $\mathcal{R}_{Q}$ seen in Figs. 2(g) and 2(h) vanishes if $\kappa_{21}=\kappa_{31}$ and $\Gamma_{2}=\Gamma_{3}$. Indeed it is known [6] that Eqs. (1) give symmetric solutions for the two modes $|2\rangle$ and $|3\rangle$ under these conditions.

\section{SELF- AND CROSS-PHASE-MODULATION}

In addition to the above third-order stimulated Raman terms, other nonlinear optical effects can affect the control scenario. These include self- and cross-phase-modulation (SPM and XPM). SPM, a resonant four-photon $\chi^{(3)}$ effect, is known to have occurred in the Raman experiments $[2,16]$, and has been known to accompany picosecond-pulsegenerated stimulated Raman scattering for many years [17].

The terms leading to SPM are shown in polarization diagrams in Fig. 3(a). The quantum system starts and ends in (a) SPM

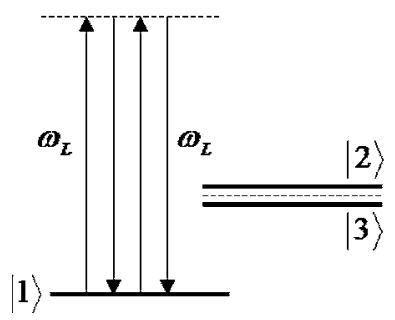

(b) XPM

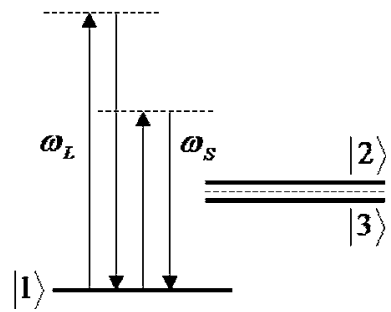

FIG. 3. (a) Example diagram leading to self-phase-modulation of the pump pulse $E_{L}(z, t)$. (b) Example diagram leading to crossphase-modulation of the Stokes pulse $E_{S}(z, t)$ by the pump pulse $E_{L}(z, t)$.

level $|1\rangle$, but the intermediate states are all off resonant. Similar diagrams involving only $\omega_{S}$ are needed to describe the SPM of the Stokes pulse. Along with SPM of the pump and the Stokes pulses, we also include the corresponding four-photon cross-phase-modulation terms between the pump and Stokes pulses of the type shown in Fig. 3(b), plus all associated time-ordering permutations.

The result of including SPM and XPM is the inclusion of terms in Eqs. (1a) and (1b) that are proportional to $\left|E_{L}(z, t)\right|^{2}$ and $\left|E_{S}(z, t)\right|^{2}[11]$. That is,

$$
\begin{aligned}
\frac{\partial}{\partial z} E_{L}(z, t)= & i \eta_{L} E_{S}(z, t)\left[\kappa_{21} Q_{21}(z, t) e^{-i(\Delta / 2) t}\right. \\
& \left.+\kappa_{31} Q_{31}(z, t) e^{i(\Delta / 2) t}\right]+i \frac{\omega_{L} n_{2}}{c}\left[\left|E_{L}(z, t)\right|^{2}\right. \\
& \left.+2\left|E_{S}(z, t)\right|^{2}\right] E_{L}(z, t), \\
\frac{\partial}{\partial z} E_{S}(z, t)= & i \eta_{S} E_{L}(z, t)\left[\kappa_{21} Q_{21}^{*}(z, t) e^{i(\Delta / 2) t}\right. \\
& \left.+\kappa_{31} Q_{31}^{*}(z, t) e^{-i(\Delta / 2) t}\right]+i \frac{\omega_{S} n_{2}}{c}\left[2\left|E_{L}(z, t)\right|^{2}\right. \\
& \left.+\left|E_{S}(z, t)\right|^{2}\right] E_{S}(z, t),
\end{aligned}
$$

where $n_{2}$ is the nonlinear index of refraction. An expression for $n_{2}$, analogous to those for the $\kappa_{i j}$ constants, could be derived using similar steps as those outlined in, for example, Ref. [12]. However, here we simply set $n_{2}=5.14$ $\times 10^{-16} \mathrm{~cm}^{2} / \mathrm{W}$, in accord with measured estimates in the literature, which are on the order of $10^{-16}-10^{-15} \mathrm{~cm}^{2} / \mathrm{W}$ [18].

Figure 4 shows an example of the control when SPM and XPM are included. To clearly distinguish the effects of SPM and XPM from the nonlinear effects introduced by the saturated Raman coupling of Sec. II, we eliminate the latter by setting the mode parameters equal, with $\kappa_{21}=\kappa_{31}=1.8$ a.u. and $\Gamma_{2}=\Gamma_{3}=22.6 \mathrm{~cm}^{-1}$. The propagation length $z=0.24 \mathrm{~cm}$ is chosen to be just on the edge of the saturation regime for these parameters. The resulting Stokes spectra, without SPM included (i.e., $n_{2}=0$ ), are shown in Figs. 4(a)-4(c), for a variety of phase offsets $\phi_{L}$. The triple-peaked structure previously found in the region of exponential Stokes gain is 

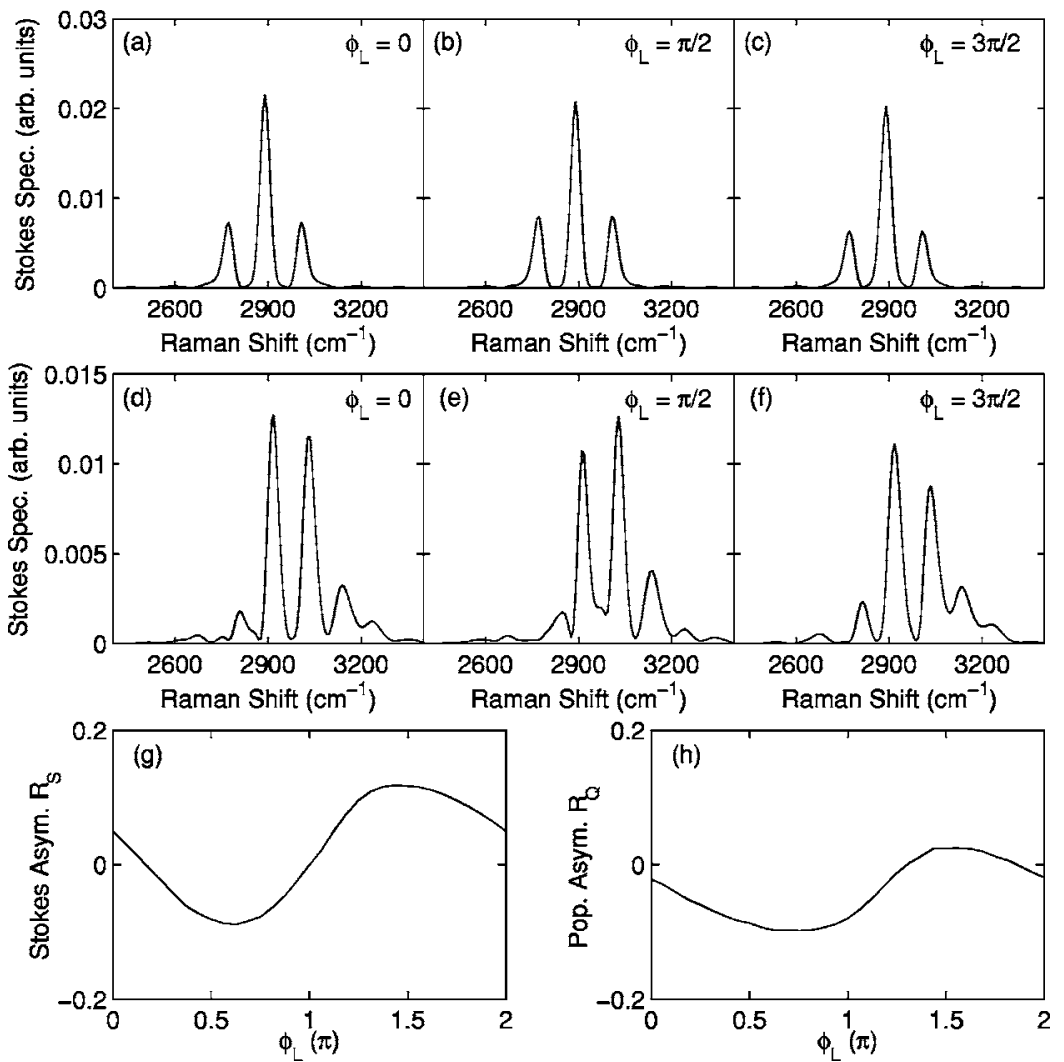

again seen and there is neither asymmetry in the Stokes spectra, nor any dependence on $\Phi_{L}$. By contrast, panels (d)-(f) plot results for the same cases as (a)-(c), but with SPM and XPM included (i.e., with $n_{2}=5.14 \times 10^{-16} \mathrm{~cm}^{2} / \mathrm{W}$ ). The Stokes spectra have now lost the triple-peaked structure and more closely resemble the two-peaked spectra observed in the experiments [5]. Furthermore, the ratio of peak heights is found to depend on $\phi_{L}$. The onset of sideband generation driven by SPM and XPM is also observed. Such sideband generation has been seen in SPM studies [19] of the same two Raman modes of methanol considered here.

The full dependence of the Stokes mode asymmetry $\mathcal{R}_{S}$ on $\phi_{L}$, with SPM and XPM included, is shown in Fig. $4(\mathrm{~g})$. A clear sinusoidal dependence of $\mathcal{R}_{S}$ on $\phi_{L}$ of similar amplitude as that seen in the experiment [5] is evident (but again the frequency of modulation with respect to $\phi_{L}$ is half that reported in the experiment). Although the population asymmetry $\mathcal{R}_{Q}$, shown in Fig. 4(h), in the scenario using SPM and $\mathrm{XPM}$ has a similar sinusoidal dependence on $\phi_{L}$ as $\mathcal{R}_{S}$, the absolute asymmetry of the populations does not match that of the Stokes lines.

It is difficult to estimate the relative importance of the SPM- and XPM-induced control as compared to the saturated stimulated Raman scattering mechanism discussed in Sec. II. Both mechanisms are nonlinear effects and depend sensitively on the pump pulse intensity and the nonlinear coupling parameters $\left(n_{2}, \kappa_{1 i}\right)$. A much more detailed theoretical study (for example, realistic calculations of the coupling parameters) and/or additional input from the experiment (for example, the spectrum of the output pump pulse) would be required to definitively address the relative contribution of each mechanism.
FIG. 4. Spectrum of the Stokes emission with and without self-phase-modulation for the case of equal Raman mode parameters. The top row (a),(b),(c) shows Stokes spectra, without SPM included, just on the edge of pump saturation $(z=0.24 \mathrm{~cm}$ for the current Raman mode parameters), for the three phase offsets $\phi_{L}=0, \pi / 2$, $3 \pi / 2$. The middle row (d),(e),(f) shows the same Stokes spectra including SPM. (g) shows the Stokes mode asymmetry $\mathcal{R}_{S}$ as a function of the phase offset, while (h) plots the population asymmetry $\mathcal{R}_{Q}$, both for the case with self-phase modulation included.

\section{FOCUSED-BEAM EFFECTS}

The terms leading to SPM and XPM also induce selffocusing (and cross-focusing) of the pump and Stokes pulses. The experiments [5] were done with a focused beam. Near the focus and highest intensities the strong pump will create an effective intensity-dependent lens due to the SPM and XPM terms, leading to additional focusing and defocusing of the frequency components within the pulse bandwidth. Changing $\phi_{L}$ changes the location of the fringes $\cos [(\Delta / 2) t$ $\left.+\phi_{L} / 2\right]$ relative to the total envelope $\exp \left[-4 \ln 2(t / \sigma)^{2}\right]$, and hence changes the nature of the intensity-dependent lens created by self- and cross-focusing effects. These nonlinear focusing effects could easily induce asymmetry in the spectral amplitudes by, for example, preferentially scattering either the lower or higher frequencies away from the highestintensity region. Furthermore, other third-order nonlinear effects are also known to be sensitive to the focused-beam geometry. For example, third-harmonic generation performed with focused Gaussian beams leads to asymmetry in the phase-matching conditions as a function of wave vector $\vec{k}$ [11]. This asymmetry in $\vec{k}$ then causes asymmetry in the resulting frequency spectrum.

In light of the fact that several third-order nonlinear effects can be affected by a focused beam geometry, and since the basic Raman coupling in Eqs. (1) is also a third-order nonlinear effect, it is worth looking for possible focused beam effects in the saturated regime of stimulated Raman scattering. To this end, we carried out simulations using the paraxial wave equation [11] version of Eqs. (1) 


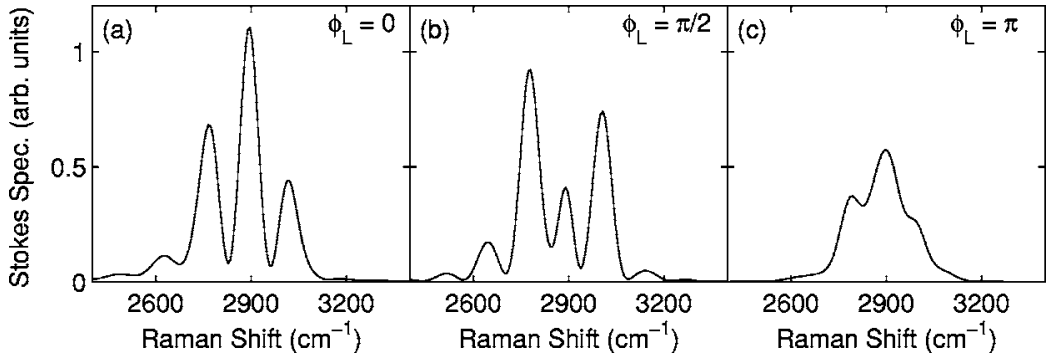

FIG. 5. Spectrum of the Stokes emission using a focused-beam geometry, for the case of equal Raman mode parameters, and for the three phase offsets $\phi_{L}=0, \pi / 2, \pi$.

$$
\frac{\partial}{\partial z} E_{n}(z, t) \rightarrow \frac{\partial}{\partial z} E_{n}(z, r, t)+\frac{1}{2 i k_{n}} \nabla_{\mathrm{T}}^{2} E_{n}(z, r, t)
$$

where the transverse Laplacian $\nabla_{\mathrm{T}}^{2}$ has been introduced to accommodate a nonplanar transverse profile of the beam. The focused-beam calculations were carried out in cylindrical coordinates where, due to cylindrical symmetry about the propagation direction $z$, the transverse Laplacian reduces to $\nabla_{\mathrm{T}}^{2}=(1 / r)(\partial / \partial r)(r \partial / \partial r)$. The Crank-Nicholson method [20] was used to numerically propagate the effects of the transverse Laplacian $\nabla_{\mathrm{T}}^{2}$ along $z$; lower-order methods, such as Euler's method or Verlet's method, were found to be unstable for this problem.

An example of Stokes spectra using the focused beam geometry is shown in Fig. 5. Again to separate the effects of the focused beam geometry from the previous control mechanisms related unequal Raman mode parameters and SPM and XPM, we chose equal mode parameters: $\kappa_{21}=\kappa_{31}=2.375 \mathrm{a} . \mathrm{u}$. and $\Gamma_{2}=\Gamma_{3}=22.6 \mathrm{~cm}^{-1}$, and exclude SPM and XPM (i.e., $n_{2}=0$ ). The initial temporal shape of the pulse was double Gaussian [Eq. (5)] with $E_{0}=0.0017$ a.u. $\left(10^{11} \mathrm{~W} / \mathrm{cm}^{2}\right)$ and $t_{p}=0.5 \mathrm{ps}$. The initial radial dependence of the pump was matched to the incoming Gaussian beam solution to the paraxial wave equation [11]. To model the experiment we note that the pump pulse was experimentally focused into a $10 \mathrm{~cm}$ cell containing liquid methanol using an $f=40 \mathrm{~cm}$ focal length lens. Assuming a typical beam diameter before the focusing lens of $D=0.5 \mathrm{~cm}$, and a diffraction-limited (i.e., ideal) focus diameter $d=0.44 f \lambda / D$, where $\lambda$ is the pump wavelength, gives a Gaussian beam waist $w_{0}$ required for the Gaussian beam formulas of $w_{0}=d / 2=14 \mu \mathrm{m}$. Simulations are started at $z=-3 b$ and propagated to $z=3 b$, where $b=2 \pi w_{0}^{2} / \lambda$ is the confocal parameter, a measure of the longitudinal width of the focal spot.

Figures 5(a)-5(c) show the resulting Stokes spectra for the three values of $\phi_{L}=0, \pi / 2$, and $\pi$. The Stokes spectra simulated using the focused-beam geometry shows asymmetry, unlike the case for the one-dimensional geometry with equal mode parameters, which would yield completely symmetric Stokes spectra. Such asymmetry was seen to appear only when there was also depletion of the pump pulse, indicating that the important symmetry-breaking component is again the nonlinear mixing of the pump and Stokes pulses. Unfortunately, the size of the focused-beam simulations restricted the size of the parameter space that could be searched, and we did not find a particular set of parameters that could produce Stokes spectra with a $\phi_{L}$ dependence that more closely matched the experimental results.

It is again difficult to gauge the relative contribution of this mechanism as compared to the previous two mechanisms identified in Secs. II and III without additional input from experiment. The details of the focal spot, and hence the nonlinear response of the stimulated Raman scattering at the high-intensity region, depend sensitively on the spatial profile of the pump pulse. This is especially true once self- and cross-focusing effects are included, which we neglected in our focused-beam simulations. Information about the spatial profile after pulse shaping was not recorded in the experiments [5]. A reliable quantitative analysis of the focusing effects in these experiments is therefore not possible.

\section{EXCITED-STATE COUPLING}

Finally, we consider the proposal of Ref. [5] that transitions between the excited states, driven by either the pump pulse alone and/or the Stokes pulse alone (Fig. 6), can account for the observed control. Here we show that such contributions are negligible.

The couplings terms corresponding to these contributions are derived in analogy with the derivation of the Raman couplings [12]. The equations of motion for the off-diagonal density matrix elements, with these terms included, are

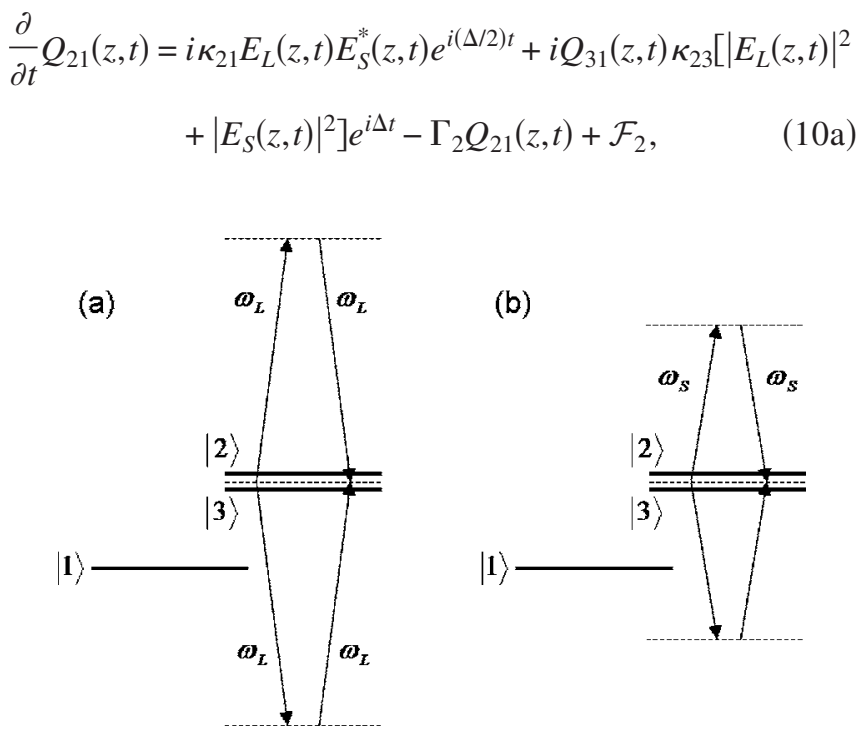

FIG. 6. Two-photon (a) laser- and (b) Stokes-driven transitions between the excited states. These transitions are all detuned by $\pm \Delta$. 


$$
\begin{aligned}
\frac{\partial}{\partial t} Q_{31}(z, t)= & i \kappa_{31} E_{L}(z, t) E_{S}^{*}(z, t) e^{-i(\Delta / 2) t} \\
& +i Q_{21}(z, t) \kappa_{23}\left[\left|E_{L}(z, t)\right|^{2}+\left|E_{S}(z, t)\right|^{2}\right] e^{-i \Delta t} \\
& -\Gamma_{3} Q_{31}(z, t)+\mathcal{F}_{3},
\end{aligned}
$$

where

$$
\kappa_{23}=\sum_{m} \mu_{2 m} \mu_{3 m}\left(\frac{1}{\omega_{m 1}-\omega_{L}}+\frac{1}{\omega_{m 1}+\omega_{S}}\right) .
$$

The lowest-order contributions that these new coupling terms make to the polarization are

$$
P_{S}^{(5)}(z, t)=P_{S 2}^{(5)}(z, t)+P_{S 3}^{(5)}(z, t)
$$

where

$$
\begin{aligned}
P_{S 2}^{(5)}(z, t)= & -N \kappa_{31}^{*} \kappa_{21} \kappa_{23} E_{L}(z, t) e^{i(\Delta / 2) t} \\
& \times e^{-\Gamma_{2} t^{t}} d t_{-\infty}^{t} e^{\Gamma_{2} t^{\prime}}\left[\left|E_{L}\left(z, t^{\prime}\right)\right|^{2}+\left|E_{S}\left(z, t^{\prime}\right)\right|^{2}\right] e^{-i \Delta t^{\prime}} \\
& \times e^{-\Gamma_{3} t^{\prime}} \int_{-\infty}^{t^{\prime}} d t^{\prime \prime} e^{\Gamma_{3} t^{\prime \prime}} E_{L}^{*}\left(z, t^{\prime \prime}\right) E_{S}\left(z, t^{\prime \prime}\right) e^{i(\Delta / 2) t^{\prime \prime}}
\end{aligned}
$$

and

$$
\begin{aligned}
P_{S 3}^{(5)}(z, t)= & -N \kappa_{31} \kappa_{21}^{*} \kappa_{23} E_{L}(z, t) e^{-i(\Delta / 2) t} \\
& \times e^{-\Gamma_{3} t^{t}} \int_{-\infty}^{t} d t^{\prime} e^{\Gamma_{3} t^{\prime}}\left[\left|E_{L}\left(z, t^{\prime}\right)\right|^{2}+\left|E_{S}\left(z, t^{\prime}\right)\right|^{2}\right] e^{i \Delta t^{\prime}} \\
& \times e^{-\Gamma_{2} t^{\prime}} \int_{-\infty}^{t^{\prime}} d t^{\prime \prime} e^{\Gamma_{2} t^{\prime \prime}} E_{L}^{*}\left(z, t^{\prime \prime}\right) E_{S}\left(z, t^{\prime \prime}\right) e^{-i(\Delta / 2) t^{\prime \prime}} .
\end{aligned}
$$

It is important to recognize that these additional contributions to the polarization are fifth order in the electric fields. At the Stokes wavelength, we would expect the fifth-order response to be negligible compared to the stimulated Raman terms, which are third-order nonlinear effects.

To demonstrate this numerically, the calculations of the previous section were repeated with the excited-state coupling terms [those leading to Eqs. (13)] included. With the excited-state coupling constant $\kappa_{23}=\left(\kappa_{21}+\kappa_{31}\right) / 2$, the results were found to change by $\sim 1 \%$. The excited-state coupling terms were found to dominate the dynamics only when $\kappa_{23}$ was about 1000 times larger than $\kappa_{21}$ and $\kappa_{31}$, a highly unphysical situation. Such a large value of $\kappa_{23}$ would be required to affect the results because the excited-state coupling terms appearing in Eqs. (10) are diminished by the offdiagonal density matrix elements $Q_{21}$ and $Q_{31}$, while the terms driving the Raman transitions, $i \kappa_{21} E_{L}(z, t) E_{S}^{*}(z, t) e^{i(\Delta / 2) t}$ and $i \kappa_{31} E_{L}(z, t) E_{S}^{*}(z, t) e^{-i(\Delta / 2) t}$, are not. (In our case, $Q_{21}$ and $Q_{31} \ll 1$, a result of small ground-state population depletion.)

We conclude that the excited-state coupling terms (Fig. 6) do not play an important role in the control of the Stokes emission.

\section{SUMMARY}

We have exposed the mechanisms underlying a recent molecular control experiment [5] in liquid methanol that showed Stokes emission control by varying the relative phase between two frequency-shifted Gaussian pulse. Specifically, we found that control of the Stokes emission is only possible, in this particular scenario, when the pump and Stokes pulses undergo coupled nonlinear optical modulations. The specific effects studied included stimulated Raman scattering in the saturated regime [9], and self- and crossphase-modulation due to the intensity-dependent refractive index [11]. It was also demonstrated that the presence of a focused-beam geometry can affect the final asymmetry seen in the Stokes spectra.

In all the mechanisms studied, the ratio of peak heights in the simulated Stokes spectra did not reflect the ratio of vibrational populations in the two Raman modes, as was previously assumed [1-5]. Moreover, quantum interference effects are not needed to qualitatively explain the control of the Stokes emission - the control can be modeled as a classical nonlinear optical effect [11] that arises once the bandwidth of the pump pulse is on the order of the energy separation of the two Raman modes. If they exist, quantum interference effects will first appear in the fifth-order polarization of the medium. However, the fifth-order contribution to the emission at the Stokes wavelengths was found to be negligible as compared to that of third-order stimulated Raman scattering.

We also included SPM and XPM into the focused-beam simulations, but these results can be extended. Specifically, we could not sample enough of the large parameter space to characterize the various nonlinear effects. In general, however, the Stokes spectra were found to depend quite sensitively on the relative strength of all the nonlinear terms. Part of the sensitivity comes from the overlapping thresholdlike behavior of the various nonlinear effects and part from the numerical instabilities of the low-order numerical methods used to propagate the Raman coupling and SPM and XPM terms that appear if the numerical method is pushed deeper into the strongly driven regime, for example, when using longer propagation length or larger pump pulse intensities. More advanced numerical techniques [20,21] would need to be implemented in order to study farther into this regime.

In conclusion, competing nonlinear optical effects may often be important when interpreting strong-field laser control experiments in liquids. Due to the large number densities, the strong nonlinear response of the liquid medium may cause significant changes in the pump pulse, which could then affect the underlying molecular control scenario.

\section{ACKNOWLEDGMENTS}

M.S. would like to acknowledge financial support from the National Science and Engineering Research Council of Canada (NSERC). This work was supported by NSERC and by Photonics Research Ontario. 
[1] T. C. Weinacht, J. L. White, and P. H. Bucksbaum, J. Phys Chem. A 103, 10166 (1999).

[2] B. J. Pearson, J. L. White, T. C. Weinacht, and P. H. Bucksbaum, Phys. Rev. A 63, 063412 (2001).

[3] T. C. Weinacht and P. H. Bucksbaum, J. Opt. B: Quantum Semiclassical Opt. 4, R35 (2002).

[4] J. L. White, B. J. Pearson and P. H. Bucksbaum, J. Phys. B 37, L399 (2004).

[5] B. J. Pearson and P. H. Bucksbaum, Phys. Rev. Lett. 92, 243003 (2004).

[6] M. Spanner and P. Brumer, preceding paper, Phys. Rev. A 73, 023809 (2006).

[7] M. Shapiro and P. Brumer, Principles of the Quantum Control of Molecular Processes (John Wiley \& Sons, Hoboken, NJ, 2003).

[8] S. A. Rice and M. Zhao, Optical Control of Molecular Dynamics (John Wiley \& Sons, New York, 2000).

[9] G. I. Kachen and W. H. Lowdermilk, Phys. Rev. A 14, 1472 (1976).

[10] B. J. Pearson and P. H. Bucksbaum, Phys. Rev. Lett. 94, 209901(E) (2005).

[11] R. W. Boyd, Nonlinear Optics (Academic Press, San Diego, 1992).

[12] M. G. Raymer and J. Mostowski, Phys. Rev. A 24, 1980 (1981).
[13] R. L. Carman, M. E. Mack, F. Shimizu, and N. Bloembergen, Phys. Rev. Lett. 23, 1327 (1969).

[14] M. Lewenstein, Z. Phys. B: Condens. Matter 56, 69 (1984).

[15] S. E. Harris and A. V. Sokolov, Phys. Rev. A 55, R4019 (1997); , Phys. Rev. Lett. 81, 2894 (1998).

[16] P. H. Bucksbaum (private communication).

[17] S. L. Shapiro, J. A. Giordmaine, and K. W. Wecht, Phys. Rev. Lett. 19, 1093 (1967); Y. R. Shen and Y. J. Shaham, Phys. Rev. 163, 224 (1967); G. G. Bret and H. P. Weber, IEEE J. Quantum Electron. 4, 807 (1968); M. Maier, W. Kaiser, and J. A. Giordmaine, Phys. Rev. 177, 580 (1969); J. I. Gersten, R. R. Alfano, and M. Belic, Phys. Rev. A 21, 1222 (1980).

[18] E. T. J. Nibbering, M. A. Franco, B. S. Prade, G. Grillon, C. Le Blanc, and A. Mysyrowicz, Opt. Commun. 119, 479 (1995); H. K. Eaton, T. S. Clement, A. A. Zozulya, and S. A. Diddams, IEEE J. Quantum Electron. 35, 451 (1999); W. Liu, S. L. Chin, O. Kosareva, I. S. Golubtsov, and V. P. Kandidov, Opt. Commun. 225, 193 (2003).

[19] P. A. Cornelius and C. B. Harris, Opt. Lett. 6, 128 (1981).

[20] W. H. Press, B. P. Flannery, S. A. Teukolsky, and W. T. Vetterling, Numerical Recipes in C, 2nd ed. (Cambridge University Press, Cambridge, U.K., 1992).

[21] W. F. Ames, Numerical Methods for Partial Differential Equations, 3rd ed. (Academic Press, San Diego, 1992). 